\title{
INSTRUMENTS: \\ A CASE STUDY OF THE INTERFACE BETWEEN SYNTAX AND LEXICAL SEMANTICS
}

\author{
NAOYUKI ONO \\ Naruto University of Education
}

\begin{abstract}
Instruments generally fall into two classes: those which can appear as the subject of sentence and those which cannot. The difference stems from the semantic dependency between causation and instrumentality involved in the meaning of verbs. This article presents a lexical semantic analysis of instruments in relation to the mapping of semantic structure onto syntactic structure. It argues that the underlying structure of the instrumental construction is a direct structural reflection of semantic relations represented in verbs' lexical conceptual structures. Cross-linguistic evidence in support of this claim is partly drawn from the applicative construction and the serial verb construction. This account offers a challenge to a widespread assumption that the mapping between semantic and syntactic structures is mediated by argument structure.*
\end{abstract}

\section{The Problem}

In English, there is a class of verbs that exhibit an alternation in the expression of instrumental NPs that occur in two syntactic positions: the object of the preposition with and the subject of sentence:

(1) a. John opened the door with the new key.

b. The new key opened the door.

(2) a. Bill hit the horse with the stick.

b. The stick hit the horse.

(3) a. Sue cut wood with the axe.

b. The axe cut the wood.

* This article is partly based on a paper read at the 15th Annual Meeting of the Kansai Linguistic Society held at Kyoto University of Foreign Studies in November 1991. Thanks to two anonymous $E L$ reviewers for their comments and criticisms on an earlier draft. I am, of course, solely responsible for its contents.

English Linguistics 9 (1992) 196-222 -196-

(C) 1992 by the English Linguistic Society of Japan 
(4) a. Marc sliced the salami with a knife.

b. A knife sliced the salami.

As is well-known, the correspondence between the (a) and (b) sentences of (1)-(4) does not always hold for all combinations of transitive verbs and instrumental phrases:

(5) a. Bill ate the meat with a fork.

b. *The fork ate the meat.

(Levin and Rappaport (1988))

(6) a. Mira saw the crack with the magnifying glass.

b. *The magnifying glass saw the crack.

(Levin and Rappaport (1988))

(7) a. George wrote the thesis with the pen.

b. *The pen wrote the thesis.

( 8 ) a. Elmer beat the horse with the stick.

b. *The stick beat the horse.

Following Marantz (1984), let us call the former "intermediary" instruments and the latter "facilitating" instruments for the convenience of discussion. Thus, semantic instruments generally fall into these two classes. The compatibility of a given verb with either type of instrumental phrase is basically determined by the interaction of the lexical properties of the verb and those of the noun denoting an instrumental object or substance. We must make explicit the correlation between the instrumental alternation and the semantic distinction of instrumentality expressed in verbs' lexical entries.

Another question that is of crucial relevance to the problem is whether the instrumental phrase is an argument of the verb. It seems that a fairly clear distinction can be made between arguments and adjuncts; however, in fact, there is little agreement as to which PPs are adjuncts and which are actually arguments of the verb. In particular, the instrumental PP poses a difficulty in this respect. For example, Baker (1988a, b), following Marantz (1984), takes it as an argument; on the contrary, Jackendoff (1990) and Levin and Rappaport (1988) among others claim that it is an adjunct.

Whether an element is an argument of verb or an adjunct is important in the theory of linking. Most current generative theories assume that the semantic or lexical conceptual structure (henceforth, LCS) of a verb determines its syntactic structure (Marantz (1984), Rappaport and Levin (1988), Grimshaw (1990), Speas (1990), Jackendoff (1990), among others). Many (but not all) of the theories converge on the idea that mapping between semantic relations represented in the LCS and syntactic 
relations at D-structure is mediated through a level of representation called "argument structure". It is normally assumed that semantic arguments are listed in argument structure and linked (or "projected" in the recent terminology) to syntactic positions by regular linking rules, whereas adjuncts are either not listed in argument structure and hence not present at D-structure (Speas (1990)), or they are linked to syntactic positions by special adjunct rules (Jackendoff (1990)).

In this paper, I argue that the instrumental phrases in English are adjoined to $V^{\prime}$ in syntactic structure, and that the difference of the semantic relations (intermediary and facilitating) is made at the level of LCS. In particular, despite the surface similarities, intermediary and facilitating instruments are mapped onto the syntactic position by distinct modes of linking.

\section{Argument or Adjunct?}

Consider now the possibility of accounting for the difference between intermediary and facilitating instruments in terms of the argument/adjunct distinction. In particular, consider the possibility of applying "externalization" to argument structure as proposed in the earlier argument structure theory of Williams (1981). In this view, argument structure is a set of arguments of predicate represented by thematic role labels, one of which is designated as the external argument. Suppose that the intermediary Instrument is an argument of the verb while the facilitating Instrument is an adjunct. It follows then that the verbs in (1)-(4) have the argument structure in (9a) and those in (5)-(8), (9b):

(9) a. (Agent, Patient, Instrument)

b. (Agent, Patient)

The syntactic alternation of instrumental NPs in (1)-(4) is the result of the lexical rule "Externalize Instrument" applying to (9a). The rule derives the argument structure (10) which corresponds to the (b) sentences in (1)-(4):

(10) (Agent, Patient, Instrument)

Now the reason why the externalization of Instrument fails to apply to (9b) - in other words, the instrumental alternation does not occur in (5)(8) -is straightforward: it does not apply simply because the argument structure contains no Instrument.

Notice that this account makes a prediction about the syntactic status of 
the instrumental phrases. Under the assumption that the argument structure of a predicate is directly mapped onto the D-structure-this assumption is in fact widely accepted in various theories of argument structure (Levin and Rappaport (1988), Grimshaw (1990), Speas (1990)), the argument/ adjunct distinction made at the level of argument structure should be reflected in syntax. See if we can find such syntactic differences between the intermediary and facilitating instrumental phrases.

Consider first the anti-reconstruction effect. It has been noted that there is a difference in the way that Condition $\mathrm{C}$ of the binding theory applies to wh-phrases with r-expressions in them. See (11):

(11) a. $* \mathrm{He}_{\mathrm{i}}$ destroyed those pictures of $\mathrm{John}_{\mathrm{i}}$.

b. ${ }^{*} \mathrm{He}_{\mathrm{i}}$ destroyed those pictures near $\mathrm{John}_{\mathrm{i}}$.

c.?*Which pictures of $\mathrm{John}_{\mathrm{i}}$ did $^{*} \mathrm{e}_{\mathrm{i}}$ destroy?

d. Which pictures near $\mathrm{John}_{\mathrm{i}}$ did he $_{\mathrm{i}}$ destroy?

What is at issue here is the contrast between (11c) and (11d). In (11c) the rexpression John is a complement of picture while in (11d) near John is not. From this observation, Speas (1990) suggests, following the basic idea of Lebeaux, that coreference is possible only if the r-expression is in a fronted adjunct. (11d) is acceptable since the r-expression is in the adjunct near phrase. In contrast, (11c) is ruled out because John is the argument of picture. Thus, it turns out that there is a striking argument/adjunct asymmetry in the anti-reconstruction effect. Moreover, Speas claims that not all adjuncts show anti-reconstruction effects. A distinction has to be made between the adjuncts which are assigned thematic roles from the verbs (i.e. "theta-marked adjuncts") and those which are not (i.e. "pure" adjuncts). She has found that only the latter exhibit anti-reconstruction effects.

If we accept Speas's proposal, then we will be able to use the effect of anti-reconstruction as a diagnosis for distinguishing arguments and thetamarked adjuncts from pure adjuncts. Under the assumption that Instruments are distinguished into arguments and adjuncts, we would expect that intermediary and facilitating Instruments differ with respect to antireconstruction effects. However, this is not the case as shown in (12):

(12) a. ${ }^{*} \mathrm{He}_{\mathrm{i}}$ signed the traveler's check with $\mathrm{John}_{\mathrm{i}}$ 's pen.

(facilitating)

b. ${ }^{*} \mathrm{He}_{\mathrm{i}}$ opened the door with $\mathrm{John}_{\mathrm{i}}$ 's key. (intermediary)

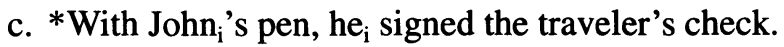

d. *With John ${ }_{\mathrm{i}}$ 's key, he $\mathrm{e}_{\mathrm{i}}$ opened the door. 
Both intermediary and facilitating instruments fail to obtain the antireconstruction effect. This fact implies that the instrumental phrases are either theta-marked adjuncts or arguments, but not pure adjuncts.

Levin and Rappaport (1988) have provided evidence which appears to be contradictory to the analysis given above. They claim that instrumental phrases are "sentential" PPs (this means in our terms that they are pure adjuncts). Their argument is based on the sentence in (13) and the sentences in (14) which are derived via the so-called do so substitution:

(13) Bill loaded the truck with cartons with a crane.

(14) a. *Bill loaded the truck with cartons and Don did so with trunks.

b. Bill loaded the truck with a crane and Don did so with a forklift.

The with phrases in (13) bear different thematic roles: with cartons has what they call the Locatum role and with a crane has the Instrument role. From the contrast in (14), they have concluded that the Locatum is an internal argument of the verb, but the Instrument is not-it is outside the VP the verb heads; hence, it is a "sentential" PP.

However, it should be noted that the do so substitution only singles out the elements that appear under the $V^{\prime}$ node. Contrary to Levin and Rappaport's claim, the effect of the do so substitution in (14b) does not necessarily entail that the instrumental phrase is outside the VP although it surely entails that it is not within the $\mathrm{V}^{\prime}$. Therefore, the structure of (13) may be something like (15):

(15) Bill [[loaded the truck with cartons $]_{\mathrm{V}}$, with a crane $]_{\mathrm{VP}}$ In this structure, the instrumental phrase is internal to the VP.

Notice that (15) is consistent with Speas' claim that Instrument is a thetamarked adjunct. The do so substitution only distinguishes arguments from non-arguments. Recall that Speas' analysis does not decide whether the instrumental phrase is a theta-marked adjunct or an argument. But, by applying the do so substitution test to the constructions including intermediary and facilitating instrumental phrases, we can make this point clear. Observe the following sentences:

(16) a. John signed a traveler's check with a Mont Blanc and Bill did so with a Parker.

b. John opened the door with his own key and Bill did so with the skeleton key.

These sentences in effect show that neither intermediary nor facilitating 
instrument is the internal argument of the verb. Thus, unlike the Locatum phrases in (14a), the instrument phrases are outside $V^{\prime}$, adjoined to that node.

In short, we have argued against the claim that the intermediary/ facilitating distinction of instrumental phrases is made at argument structure, by demonstrating that both of them show virtually no difference with respect to the syntactic effects obtained from the reconstruction and the do so substitution. ${ }^{1}$ We have shown that Instruments are neither internal arguments nor pure adjuncts. They are, as Speas calls them, theta-marked adjuncts, which are thematically related to the verb and syntactically realized within the VP. Thus, the distinction of the semantic relations in question is not directly mapped onto the syntactic structure; rather, it is made at a certain level of semantic representation, which is independent of syntactic argument structure. The representation has to predict the possibility of instrumental alternation we have seen in the first section.

\section{The Lexical Conceptual Structure}

Recent work on argument structure theory in general assumes that thematic roles are "abstractions over the finer-grained semantic structures of verbs" (Bresnan and Kanerva (1989: 24)). That is, roles such as Agent, Patient, Goal, or Instrument are not independently existing primitives; rather, they are relations which are derived from the LCS of predicates. In this section, I present an analysis the LCSs of verbs that cooccur with the intermediary and facilitating instrumental phrases. My proposal is essentially based on the semantic structure theory elaborated by Jackendoff (1990) but some modifications will be added.

Consider first the difference of the semantic properties between the two instruments. Marantz (1984) states, rather informally, as follows:

a. Elmer unlocked the porcupine cage with a key.

${ }^{1}$ Baker (1988a: 242-243) shows, based on some effects of the ECP, that benefactive, instrumental, and locative phrases are arguments of verbs. He notes: "it does not necessarily follow from the fact that benefactive and instrumental phrases are never obligatory that they are not theta-marked by the verb when they do appear" (p. 239). His claim is compatible with Speas' view (and the view I am adopting here) that thetamarked adjuncts are adjoined to $\mathrm{V}^{\prime}$. 
b. Elmer examined the inscription with the magnifying glass.

In (7.43a) $a$ key is an intermediary agent in the act of unlocking the porcupine cage; Elmer does something to the key, the key does something to the cage, and the cage unlocks. In (7.43b), the magnifying glass is an indispensable tool in Elmer's examination of the inscription, but it is not an intermediary agent in the examination. (p. 247)

Assuming that the intuitive content of this statement is basically correct (see also Jackendoff (1990), Pinker (1989)), I propose the following conceptual structure representation in (18) for the verb open that appears in the sentence in (17):

(17) John opened the door with the key.

$\left[\begin{array}{l}\text { CAUSE([JOHN], }\left[\begin{array}{l}\text { CAUSE([KEY], [INCH([BE([DOOR }], \\ [\mathrm{OPEN}])]) \text { AFF }([\mathrm{KEY}],[\mathrm{DOOR}])\end{array}\right] \\ \mathrm{AFF}([\mathrm{JOHN}],[\mathrm{DOOR}])\end{array}\right]$

The topmost event in this conceptual structure represents the spatial motion and location constituting the verb's meaning - this part is called "thematic tier" (Jackendoff (1990: 126)). This semantic representation in effect describes a causal chain in which the superordinate event including an initiating cause (an agent or instigator of the event) contains a sub-event including an instrument, which, in turn, is in a causative relation resulting in the final event. Thus, the whole event is construed as " $\mathrm{X}$ causes $\mathrm{Z}$ which causes $Y$," which is cognitively equivalent to " $X$ causes $Y$ by means of $Z$ ". The function AFF, appearing in the subordinate events (called "action tiers"), represents Actor-Patient relations (see Jackendoff (1990: Ch.7) for more discussion).

(18) is the maximally-specified semantic representation, corresponding to the transitive form of the verb, as in (17). To relate it to the intransitive (i.e. unaccusative) form of the verb, Jackendoff (1990: 71-76, 249-255) introduces a means of representing optional parts of LCS (what he calls "lexical abbreviation notations"). The transitive and intransitive lexical forms are related by specifying the optionality of the outer function CAUSE and its first argument. (He uses dashed underlines for indicating optional part of conceptual constituent.) Following his basic idea, I propose

${ }^{2}$ See Foley and Van Valin (1984: 53-55) and Pinker (1989: 198-199) for similar but slightly different views on the causal link involved in the instrumental construction. 
to extend the notion of lexical abbreviation slightly. Incorporating this elaboration yields a fuller conceptual structure representation for open, which iterates the dashed underline notation as illustrated in (19), and corresponds to the following paradigm of syntactic forms of open in (20):

(19) [CAUSE ([JOHN], [CAUSE ([KEY], [INCH ([BE ([DOOR], [OPEN])])])]

a. John opened the door with the key.
b. The key opened the door.
c. The door opened.
d. The door is open.

There are four related LCSs abbreviated to (19); each of them corresponds to the (a), (b), (c), or (d) sentence in (20). On this account, it is not necessary to designate one of the arguments in argument structure as external. The external argument is simply the most "prominent" semantic argument in the verb's LCS. ${ }^{3}$ At the moment we will not discuss any more details of syntactic realization of the LCS. We will return to this topic in the following section.

In contrast to the LCS of intermediary instrument, I suggest that the LCS representation of the verbs that cooccur with the facilitating instrument does not have the instrumental argument involved in the main causative relation; rather, the instrument is an argument of the modifying sub-event clause. I tentatively propose to postulate the function $\mathrm{USE}^{4}$ that relates the Actor and the Instrument in the modifier clause:

$\left[\begin{array}{l}\text { CAUSE ([ JOHN], [GO ([PASTA], [TO [IN [MOUTH OF } \\ {[\text { JOHN]]]])])) [BY [USE ([JOHN], [FORK])]] }}\end{array}\right]$

I assume that the means clause is related to the thematic tier by the function BY, which, according to Jackendoff (1990: 142), turns events into means modifiers. Alternatively, it may be possible to assume that the subevent is in a causal "subordinating relation" to the main event (see Pinker (1989: 196-200)). Whatever the relation may be, it is important to note that the Instrument does not form a causal chain with the Actor and the Patient.

This explains why instrumental alternation does not occur to facilitating

${ }^{3}$ Cf. Grimshaw's (1990) proposal that the external argument is an argument that is maximally prominent in both aspectual and thematic dimensions of argument structure.

${ }^{4}$ The function USE may be decomposed into some more primitive functions. I leave this issue open here. See Jackendoff (1990: 259). 
instruments. As we have seen in (19) and (20), the intermediary instrument is the external argument in the LCS corresponding to (19b). This option is not available in (21); the facilitating instrument, being an argument in the adjunct clause, cannot be mapped onto the subject position.

An immediate consequence of our account is the prediction that intransitive (unergative) verbs do not take intermediary instrumental phrases because, by assumption, intermediary instruments exist in conceptual structure in relation to Agents and Patients. Thus, without a causal chain in which the intermediary instrument appears, the instrumental phrases cooccuring with intransitive verbs are facilitating instruments. Therefore, this account predicts that instrumental subjects do not occur with intransitive verbs. To the best of my knowledge, this prediction is true:

(22) a. She was running with new shoes.

b. *The new shoes ran.

(23) a. Alex swam across the river with flippers.

b. *The flippers swam across the river.

(24) a. The man walked with a cane.

b. *The cane walked.

It should be noted that the analysis presented here is not the same as what Jackendoff (1990) proposes for the role Instrument. ${ }^{5}$ In his analysis, instrument is a conceptual constituent that appears in a means expression ([BY [...]]) and is linked to the syntactic phrase with NP by an adjunct rule. He writes:

... so-called Instrumental subjects such as the key in The key opened the door probably do not fall under this analysis. I am inclined rather to regard them as inanimate Instigators-that is, not as grammatical Instrument at all. (p. 295, fn. 8)

However, in order to account for the correlation of instrumental subjects and the semantic distinction of instruments, I believe that the verbs correspond to the distinct LCS representations as proposed on our account.

5 Jackendoff suggests the following LCS for the verb in Phil opened the door with a key (p.142):

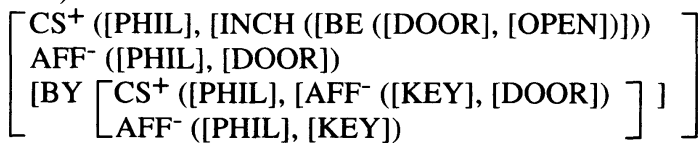

His LCS differs from ours in that the instrumental argument is not included in the thematic tier. 
An argument in favor of our approach is formulated in relation to the derivation of instrumental -er nominals. Levin and Rappaport (1988) observe that instrumental -er nominals are derived from the verbs that cooccur with intermediary instruments. It is normally the case that -er nominals such as baker, singer, killer in general refer to the external argument of their base verbs. Levin and Rappaport have pointed out a contrast between the nominals in (25) which are derived from the verbs in (1)-(4) and the nominals in (26) based on the verbs in (5)-(9):

(25) opener, cutter, slicer, sander

(26) eater, seer, writer, beater

Namely, those in (25) denote either Agent or Instrument; those in (26) do not bear the instrumental reading, i.e., they may refer to Agent but not to Instrument. ${ }^{6}$ On our account, this contrast follows immediately from the difference of the LCSs suggested here. The base verbs of the nominals in (25) correspond to two LCSs: one with the external Agent and one with the external Instrument. The base verbs of the nominals in (26), on the other hand, correspond to one LCS representation. Thus, the external Instrument is not available. I believe that the difference of interpretation of the nominals in (25) and (26) strongly suggests that the instrumental argument is qualified as an external argument in the lexical representation of the former but not in the lexical representation of the latter. Note that Levin and Rappaport claim that the instrumental -er nominals, which they call "nonevent nominals", do not "inherit" argument structures of their base verbs (see also Grimshaw (1990) for a similar treatment of derived nominals). Since our theory defines the external argument at LCS without recourse to argument structure, it is consistent with Levin and Rappaport's or Grimshaw's view that the instrumental nominals, and nonevent nominals in general, lack argument structures.

\section{Mapping from LCS to D-Structure}

Having laid out the conceptual structures of the instruments, we are now in a position to discuss how they are associated with the syntactic structures. We have argued earlier that the instrumental with phrases are

${ }^{6}$ Some compounds based on these nominals may refer to instruments, e.g. a typewriter, an egg-beater. Thus, there may be some factors, other than the LCS, involved in the reading of these nominal compounds. But they are unclear to me at present. 
adjoined to $\mathrm{V}^{\prime}$ in phrase structure. The last section argued that the decomposed semantic structures provide the basis for an account of the difference of semantic relations holding between the verbs and the instrumental NPs. My proposal was that the difference stems from the distinct structural configurations in LCS: the intermediary instrument is a semantic argument involved in the causative event, whereas the facilitating instrument is an argument in the sub-event which itself is a modifier of the central event. Thus, in our approach, the intermediary and facilitating instruments are in the same syntactic position but are distinct at the level of conceptual structure. In what follows, I will give an account of syntactic realization (or linking) of those distinct LCS relations.

Most theories of argument linking normally invoke a general principle relating an ordered list of thematic roles (thematic hierarchy as it is often called) to an ordered list of syntactic relations (syntactic hierarchy). Various versions of thematic hierarchy can be found in the literature (Fillmore (1968), Larson (1988), Baker (1989), Bresnan and Kanerva (1989), Grimshaw (1990), Jackendoff (1990), among others). They primarily differ in what thematic roles are listed in the hierarchy and how they are ordered.

These linking theories assume (explicitly or implicitly) that the prominence relations represented in the thematic hierarchy are simply a reflection of the prominence relations expressed in lexical-semantic representations. Following this idea, the LCS analysis given in the last section will lead us to posit the thematic hierarchy: Agent > Instrument > Patient. This relative order of the relevant semantic arguments is significantly compatible with the hierarchies proposed by Fillmore (1968), Baker (1989), and Bresnan and Kanerva (1989) for independent reasons.

In Jackendoff's (1990) theory of linking, semantic arguments of a predicate are linked to syntactic positions by a general linking principle which is formulated in terms of a thematic hierarchy. On the other hand, adjuncts are subject to adjunct rules, which assure correspondences between LCS constituents and particular syntactic phrases.

Since Jackendoff takes the instrument as appearing in an adjunct clause in conceptual structure, it is assumed to be linked to the syntactic position by an adjunct rule. This rule is called the Instrumental Adjunct Rule. ${ }^{7}$ I

7 The rule is formulated as follows (Jackendoff (1990: 188)): 
will adopt his proposal that adjuncts are linked by adjunct rules; namely, facilitating instruments are realized successfully as the the syntactic with phrase by means of the Instrumental Adjunct Rule.

However, my proposal crucially differs from Jackendoff's in that intermediary Instruments are actually arguments of verbs. Thus, under the present account I want to suggest that instrumental arguments are linked in a principled way. In this regard, I will appeal to a proposal by Larson (1988).

Larson probably makes the strongest claim among the current linking theories. The basic assumption, which follows the idea of Baker's (1988a) Uniformity of Theta Assignment Hypothesis, is that relative prominence of thematic roles in the hierarchy are directly mapped onto relative structural positions in syntax. This is explicitly stated in his mapping principle:

If a verb a determines $\theta$-roles $\theta_{1}, \theta_{2}, \ldots, \theta_{n}$, then the lowest role in the Thematic Hierarchy is assigned to the lowest argument in constituent structure, the lowest role to the next lowest argument, and so on.

(Larson (1988: 382))

Assuming the thematic hierarchy "Agent $>$ Theme $>$ Goal $>$ Obliques (manner, location, time, ...)", he proposes the VP structure for sentences like (27):

(27) Bill sent a letter to Mary.

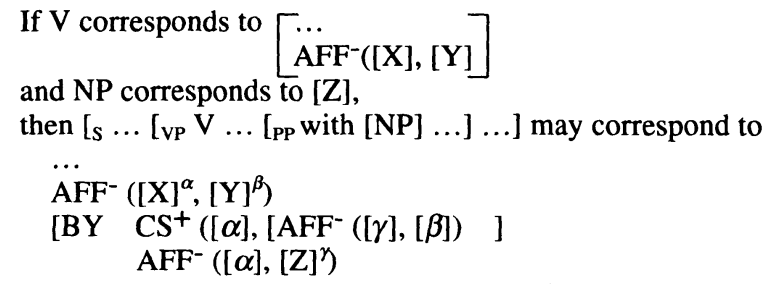

This formulation needs some adjustment so that we can use it with the LCS representation given above. 
(28)

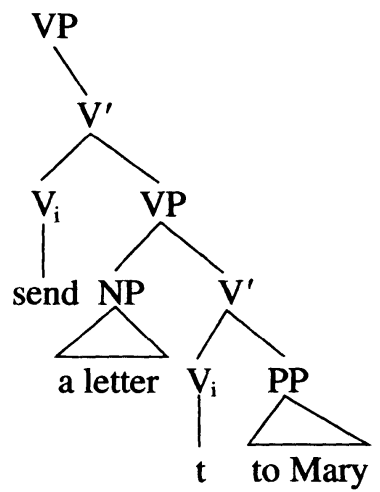

The verb moves out of the lower verb position to the head of the higher VP and assigns Case to the direct object. The double-object version of the sentence in (27) is derived from the same underlying structure, but this time the specifier of VP is "demoted":

(29) Bill sent Mary a letter.

(30)

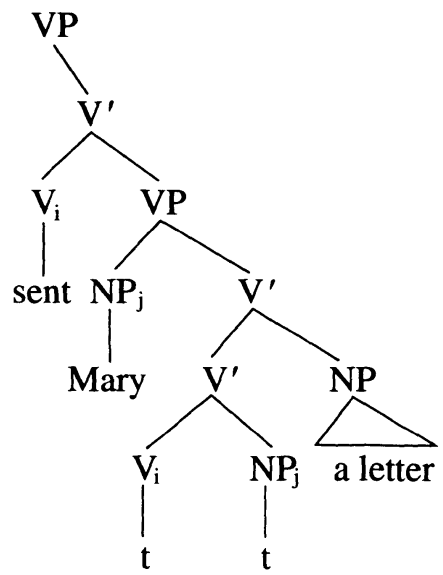

As the result of demotion, the indirect object Mary moves up to the vacated SPEC position and receives Case from the raised verb. A letter, being adjoined to the $\mathrm{V}^{\prime}$, receives an inherent Case.

Turning now to the instrumental constructions, I propose that the sentence in (31) derives in exactly the same fashion as the double-object construction derives through the demotion and the V-movement: ${ }^{8}$

${ }^{8}$ Larson does not explicitly state that instruments are involved in his thematic hierarchy. See Baker (1989: 544, fn. 23) and Speas (1990: 79). 
(31) John opened the door with a key.

(32)

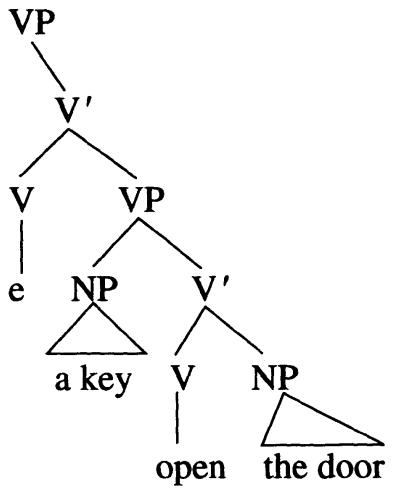

(33)

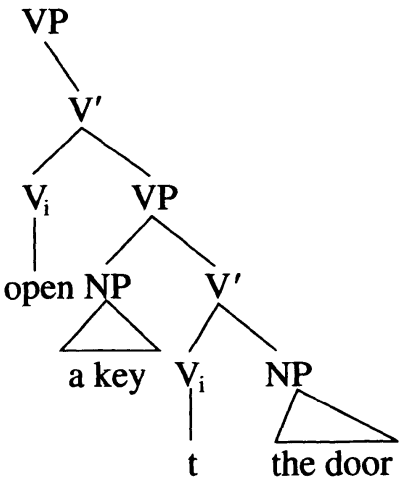

(34)

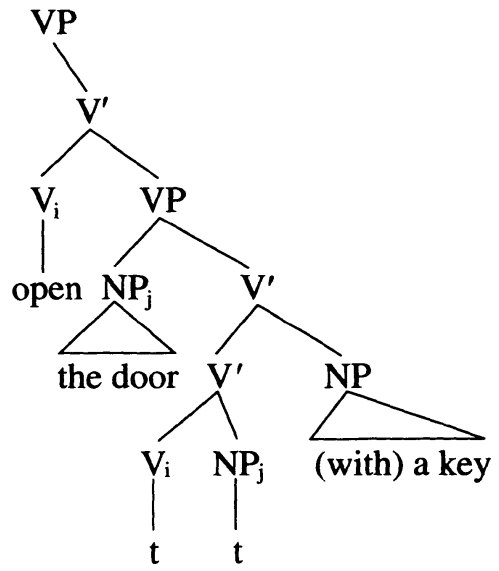

The only difference is that the V-movement in (33) yields the ungrammatical sentence in (35): 
(35) *John opened a key the door.

However, the derivation of such sentences should not be precluded in advance since, in language with so-called applicative constructions, sentences parallel to (35) are in fact admissible. Thus, we take it as an advantage of our approach. We will return to discussions of applicative constructions in the next section.

Consider now what rules out the sentence in (35) and what rules in a sentence like (36) which, as mentioned, we assume to have parallel derivational history:

(36) John sent a letter to Mary. (=(27))

The answer lies, I believe, in Case theory.

Suppose that we distinguish two types of Case: inherent Case and structural Case, as suggested in Chomsky (1986). Inherent Case is assigned to NP at D-structure if and only if the assigning head theta-marks the NP, while structural Case is assigned at S-structure independently of thetamarking. More importantly, inherent Case is assigned at D-structure and realized at $\mathrm{S}$-structure. I extend this idea by assuming, under the view that the assignment of inherent Case is associated with the direct theta-marking, that every NP that is theta-marked by a head verb must have an inherent Case at D-structure. That is, an NP may be assigned both an inherent Case at D-structure and a structural Case at S-structure. If NPs are governed and assigned structural Case by INFL or V, the inherent Cases assigned to them are realized as morphologically unmarked forms (nominative or accusative) in English; otherwise, they are realized at S-structure as Case morphemes (such as the genitive' $s$ ) or prepositional elements (such as the inserted of in derived nominals). One may regard structural Case as a realization of inherent Case; however, notice that structural Case is not always a realization of inherent Case. In other words, structural Case may be assigned to NPs that are not inherently Case-marked, since the structural Case assignment does not depend upon theta-marking. Thus, verbs can exceptionally Case-mark the subject of an infinitival clause even though they do not assign inherent Case to the subject; or verbs may assign structural Case to NP when its inherent Case is absorbed. We will discuss the absorption of inherent Case immediately below.

Given this, consider the assignment of structural objective Case. Assume that the inherent Case is realized successfully as structural objective Case if and only if it meets the "affectedness condition" in the sense of Levin and Rappaport (1986), Rappaport and Levin (1988), Lebeaux (1988), Pinker 
(1989); namely, the NP that is assigned structural Case by a verb is the affected object of the verb. I use the term affectedness here loosely to refer to a change of state or location caused by the action of agent (see the works cited above). ${ }^{9}$ The affectedness requirement explains the difference between (35) and (36). (35) is ruled out because $a$ key, which is inherently Casemarked by the verb, does not meet the affectedness condition. By contrast, the object a letter in (36) satisfies it since the verb expresses a change of location.

An argument in support of our account can be constructed in relation to the sentence like (37) where, unlike (35), the instrumental NP appears as the object:

(37) I hit the bat (against the wall).

(cf. I hit the wall with the bat.) (Pinker (1989: 105))

This sentence virtually parallels (35); however, it differs in that the object is affected (in the sense of change of location) by the action denoted by the verb. See also (38):

(38) Fred broke the teacup against the window.

(cf. Fred broke the window with the teacup.)

(Foley and Van Valin (1984:56))

In (38) the instrumental object undergoes a change of state, meeting the affectedness condition. Thus, I think the evidence suffices to establish that inherent Case is realized as objective Case if and only if it satisfies the affectedness condition. ${ }^{10}$

We have already seen the absorption of inherent Case in Larson's account of the double-object construction as in (39), which we have applied to the instrumental construction in (40):

9 Affectedness should be represented in terms of the Agent-Patient relation in LCS. See Jackendoff (1990: Ch.7) for more discussion.

${ }^{10}$ As pointed out by one of the reviewers of this article, the argument presented here does not preclude the possibility that nonaffected objects of stative verbs realize their inherent Case as objective Case at S-structure. I assume that nonaffected objects do not bear inherent Case at S-structure because of the Case-absorption applying to stative verbs. It is also pointed out that this account raises a problem with respect to the expletive object it as in I take it for granted that his theory is basically correct. I think the sentence involves a small clause [it that ...] so take assigns it a structural Case as in the ECM construction. The point here is that the affectedness requirement is imposed on the structural realization of inherent Case, not on the structural Case assignment to objects. 
(39) Bill sent $t_{i}\left[v p\right.$ Mary ${ }_{j}\left[v,\left[v, t_{i} t_{j}\right]\right.$ a letter $] \quad(=(30))$

(40) John opened $d_{i}\left[v p\right.$ the door ${ }_{j}\left[v,\left[v, t_{i} t_{j}\right]\right.$ with a key] $\quad(=(34))$

Following Larson, I assume that the inherent Case which is to be assigned to Mary is absorbed; as a result, Mary moves up to the SPEC position where it receives structural Case from the raised verb. In the same way, the door does not have inherent Case as the result of Case absorption and hence it is Case-marked structurally in the SPEC position at S-structure. Now that the inherent Cases are absorbed, the objects need not meet the affectedness condition, which we are assuming to be imposed on the structural realization of inherent Cases. ${ }^{11}$

Now let us turn to the inherent Cases assigned to a letter and a key. As noted, inherent Cases are realized at S-structure either as Case morphemes (such as the genitive 's) or as prepositional elements (such as the inserted of in derived nominals). Suppose that the realization of the inherent Case associated with a Theme argument is morphologically unmarked. This gives rise to the surface form a letter in (39). Suppose further that the inherent Case associated with an instrument argument is realized as the preposition with, as in (40). Thus, we may assume that the preposition is not present at D-structure; it is inserted in the syntax, much as the preposition of in derived nominals. ${ }^{12}$

${ }^{11}$ In the passive, both structural and inherent Cases are absorbed by the passive morpheme -en. Thus, the object NP of the verb is not assigned structural or inherent Case in the object position though it is theta-marked by the verb. I suppose that the unaccusative construction is derived lexically (see Jackendoff (1990: Ch.11)); hence, it involves no syntactic movement.

${ }^{12}$ The analysis given here may be applied to the verb fill, which shows the same paradigm of open:

(i ) Water filled the glass.

(ii )*Bill filled water (into) the glass.

(iii) Bill filled the glass with water.

(iv) The glass filled (with water).

The LCS is roughly [CAUSE ([BILL], [INCH [BE ([GLASS], [FULL OF WATER]])]])] (cf. Jackendoff (1990) and Gropen et al. (1991)). The ungrammaticality of (ii) is also ascribed to the violation of the affectedness condition. Our account is supported by Levin and Rappaport's (1988) evidence that the with phrase in (iii) and (iv) parallels the instrumental with phrase, rather than the with phrase that appears with spray/load class verb.

An anonymous reviewer has drawn attention to the fact that in Japanese the sentence which is supposedly equivalent to (ii) is acceptable:

(v) Biru-wa gurasu-ni mizu-o mitasita.

Bill-Top glass-Dat water-Acc filled 
Thus far what I have tried to accomplish is the establishment of structural parallelism in the derivation of double-object constructions and instrumental constructions. To conclude the discussion in this section, consider the motivation for assuming the extra "outer" VP whose empty head serves as the "landing site" of the lexical verb. On Larson's account, this follows from a restrictive version of $\mathrm{X}^{\prime}$ theory, which requires that each head have only one complement and only one specifier, and the requirement imposed by theta theory that a predicate project as many argument positions in syntax as thematic roles that it has in its argument structure. Accordingly, three-argument verbs like give satisfy these requirements only by projecting an extra VP that supplies an argument position for the external argument. This is illustrated as follows:

$$
\begin{gathered}
{\left[\mathrm{VP}_{\mathrm{NP}}\right]\left[\mathrm{v}^{\prime} \text { e }\left[\mathrm{VP}[\mathrm{NP}]\left[\mathrm{v}^{\prime} \text { give }[\mathrm{NP}]\right]\right]\right]} \\
\text { Agent }
\end{gathered}
$$

The idea of projecting empty verbal heads is also supported by Speas (1990); however, she rejects the reasoning that Larson uses for his account. In Speas' theory, the main motive for assuming extra verbal heads lies in the LCS theory of Hale and Keyser, in which agentive transitive verbs such as break are projected from the semantic representation which is, roughly, " $x$ cause $[y$ break]". It is this extra causative predicate that projects a complex syntactic structure. Following Speas' idea, I assume that the empty verbal heads correspond to the causative predicates (in the LCS theory that I adopt here, the CAUSE and INCH functions) in the LCS representation, roughly illustrated as follows:

(42) [vp John [v, e [vp a key [v' e [vp the door [ $v^{\prime}$ e [open] ]]] [ $\mathrm{x}$ CAUSE $[\mathrm{z}$ CAUSE [INCH [ y OPEN]]]]

'Bill filled water into the glass.'

According to Gropen et al. (1991), so-called "locative verbs" generally fall into three classes: "figure-object" verbs like pour, "ground-object" verbs like fill, and "alternator" verbs like load. There is reason to believe that mit(as) belongs to the class of alternators which show both the figure-object pattern of argument realization as in (v) and the ground-object pattern as in (vi):

( vi ) Biru-wa mizu-de gurasu-o mitasita.

Bill-Top water-Obl glass-Acc filled

'Bill filled the glass with water.'

The direct object in ( $v)$ meets the affectedness condition since the verb is associated with the figure-object LCS (see Gropen et al. (1991)). There remains the question of how the two types of LCSs are related (see Jackendoff (1990: 173-174)). 
The evidence in favor of our approach is provided by an analysis of applicative constructions and serial verb constructions, which I will present in the following section.

\section{Evidence from Other Languages}

The discussion here primarily has to do with instrumental applicative constructions. I intend to demonstrate that the arguments formulated so far regarding the mapping between conceptual structures and underlying syntactic structures are also valid for the similar constructions in languages other than English. It should be noted that at an abstract level, our approach to those constructions, including English instrumental constructions, is motivated by the following methodological assumption: if we postulate structural parallelism cross-linguistically in the underlying structure, we can account for parametric differences of the surface in terms of the grammatical principles independently established in syntax; hence, the mapping from a universal conceptual structure into a languageparticular syntax can be made maximally simple.

Baker (1988a, 1988b) has shown (convincingly to me) that applicative constructions involve incorporation of prepositions. The point is that the Preposition Incorporation applies, as other Incorporations generally do, only to arguments of verbs (e.g. Goal, Benefactive, Instrumental, Locative). In light of this generalization, the following examples from Niuean (Seiter (1979)) are interesting:

(43) a. Kua hel tuai e Sione e faloa aki e titipi haana. Perf cut Perf Erg Sione Abs bread with Abs knife his 'Sione has cut the bread with his knife.'

b. Kua hele aki tuai e Sione e titipi haana e falaoa. Perf cut with Perf Erg Sione Abs knife his Abs bread 'Sione has cut the bread with his knife.'

(44) a. Ko e tohitohi a au mogonei aki e pene foou. Pres write Abs I now with Abs pen new 'I'm writing now with a new pen.'

b. * Ko e tohitohi aki a au (mogonei) e pene foou. Pres write with Abs I now Abs pen new 'I'm writing (now) with a new pen.'

Seiter argues that in this language the Preposition Incorporation is possible 
only if the host verb is transitive. ${ }^{13}$ Thus, in (43) the transitive verb "cut" (hele) incorporates the instrumental preposition aki, but in (44) the intransitive verb "write" (tohitohi) resists the incorporation. ${ }^{14}$ This contrast makes sense in our analysis of instrumental phrases if we assume that these Niuean verbs correspond to the same conceptual structures suggested for the English equivalents. Thus, on our account, the transitive/intransitive contrast observed by Seiter follows from the intermediary/facilitating distinction made at the level of LCS, and hence the ungrammaticality of (44b) can be ascribed to Baker's incorporation analysis.

Baker's account of applicatives crucially depends on the Uniformity of Theta Assignment Hypothesis, which claims that "Identical thematic relationships between items are represented by identical structural relationships between those items at the level of D-structure" (Baker (1988a: 46)). As it stands, this hypothesis does not necessarily demand that a hierarchical order of thematic roles be mapped into a particular hierarchical relation of syntactic positions. Baker assumes that in Chichewa both of the Instrument mpeni 'knife' and the Patient mtsuko 'waterpot' are sisters to the verb in the sentence (45) and in its applicative counterpart in (46):

(45) Mavuto a- na- umb -a mtsuko ndi mpeni.

Mavuto Sp Past mold Asp waterpot with knife

'Mavuto molded the waterpot with a knife.'

(46) Mavuto a- na- umb -ir -a mpeni mtsuko.

Mavuto Sp Past mold Appl Asp knife waterpot

'Mavuto molded the waterpot with a knife.'

Notice, however, that our arguments have been based on the more rigid hypothesis that underlying structures are a direct structural reflection of the thematic hierarchy. Given this, I suggest an amendment to Baker's analysis that the instrumental applied object is in a higher structural position than the basic object. This leads us to posit a structure like (47) for the sentences (45) and (46):

13 Seiter's analysis is done within the framework of Relational Grammar. He uses the term aki-Cliticization instead of Preposition Incorporation.

${ }_{14}$ As to this transitive/intransitive asymmetry, there seems to be a parametric variation. Baker (1988b) gives a few examples from Chichewa which show that intransitive verbs do incorporate instrumental prepositions. See also Bresnan and Moshi (1990). 
(47)

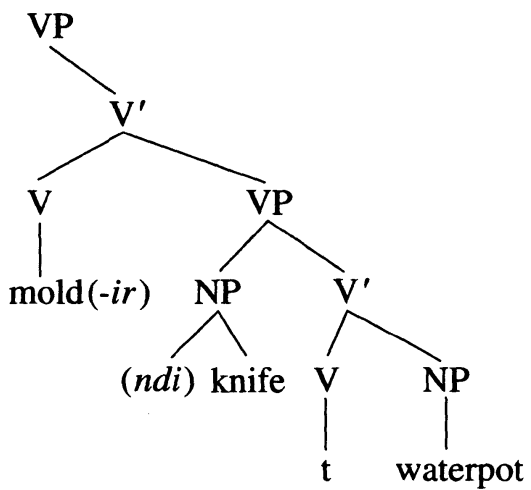

In this language the instrumental preposition ndi is affixed on the verb morphologically as the applied affix -ir; thus, these elements are in a complementary distribution. Recall that in English the structure equivalent to the applicative construction is ruled out:

(48) * John molded a knife the waterpot. (cf. (35))

We have claimed that the ungrammaticality is attributed to the violation of the affectedness condition imposed on the realization of inherent Case. Suppose that this condition holds in Chichewa as well as in English. But Chichewa differs from English in that it has the applied affix on the verb. Thus, in Chichewa the inherent Case that is to be assigned to knife can be realized as the affix -ir as well as the prepositional root ndi (Baker (1988b)). The result of realizing the inherent Case on verbs' affix is in effect the absorption of the inherent Case. Consequently, the applied object knife in (47) receives only a structural Case from the verb. Therefore, the affectedness condition does not hold in the applicative construction. English lacks the option of realizing inherent Cases as verbal affixes. Thus, sentences like (48) are rejected for the violation of the affectedness condition. The cross-linguistic difference between English and Chichewa immediately follows from our account.

A further advantage of our approach is that we can capture the structural parallelism between applicative constructions and serial verb constructions, in which "a sequence of verbs appears in what seems to be a single clause" (Baker (1989: 513)):

(49) Olè fi òbe gún oba. (Yoruba: Baker $(1989,(44 a))$ ) thief use knife stab chief

'The thief stabbed the chief with a knife.'

I propose that (49) has the structure like (50): 
(50)

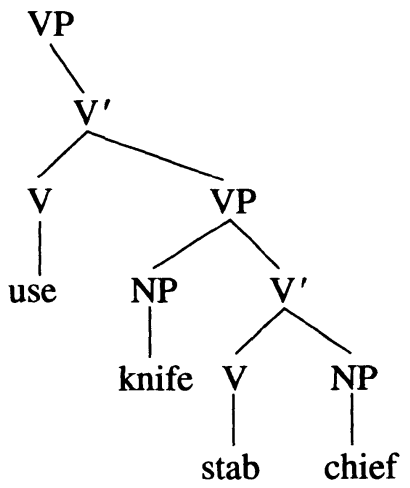

The serial verb construction differs from the applicative construction in that the higher verb is not empty. According to Foley and Van Valin (1984), in some types of the construction certain verbs are specifically used for the first verb. They note: "Cross-linguistically, the most common verbs in this usage are give, which adds benefactives or goals, and take, which adds instruments, manner NPs, and comitatives." (p. 198) Then, it would not be unreasonable to assume that $f i$ ('use' or 'take') is a grammatical realization of the empty verbal head that we have assumed in double-object constructions and instrumental constructions in English, and in applicative constructions in Chichewa.

In this regard, the examples from Yatye, cited in Foley and Van Valin (1984: 203), are suggestive. They show exactly the same paradigm as we have seen with open in English. The only difference is that in this language LCS constituents such as CAUSE (and probably INCH) correspond to the morphologically realized verb abà:

(51) a. Utsí ikù.

door close

'The door is closed.'

b. [BE ([DOOR], [CLOSE])]

(52) a. Utsí abà ikù.

door close

'The door closed.'

b. [INCH ([BE ([DOOR], [CLOSE])])]

(53) a. ìwyi abà utsí ìù.

child door close

'The child closed the door.'

b. [CAUSE ([CHILD], [INCH ([BE ([DOOR], [CLOSE])])])] 
(54) a. òtsi abà utsí ikù.

stick door close

'The stick closed the door.'

b. [CAUSE ([STICK], [INCH ([BE ([DOOR], [CLOSE])])])]

a. ìwyi abà òtsi ikù utsí.

child stick close door

'The child closed the door with a stick.'

b. [CAUSE ([CHILD], [CAUSE ([STICK], [INCH ([BE ([DOOR], [CLOSE])])])]

(56) ìwyi abà òtsi abà ikù utsí. child stick close door

'The child closed the door with a stick.'

Each of the (a) examples corresponds to the LCS (b) respectively. Although they do not show perfect one-to-one correspondences, e.g. (55) (but observe its variant (56)), it is clear that $a b a ̀$ functions as a causative verb which corresponds to the causative function in LCS.

The argument presented here is admittedly tentative and rather speculative; however, our approach implies that a more cohesive account may emerge if we assume that universal conceptual relations are mapped onto syntactic relations as directly and uniformly as possible. Of course, the applicative and serial verb constructions need more detailed parametric explanation as discussed in Baker (1988b, 1989) and Bresnan and Moshi (1990). But this topic is not our main concern in the present paper. I think many problems remain and more work is needed.

\section{Subject Hierarchy and By Phrase}

Returning now to our main concern, consider once again the linking of instrumental arguments in English. The conceptual structure of open suggested in (18), with the lexical abbreviation in (19), provides a lexicalsemantic account of Fillmore's well-known Subject Hierarchy. The hierarchy shows exactly the same order of thematic ("case" in Fillmore's terms) roles that the conceptual structure predicts:

(57) Agent $>$ Instrument $>$ Patient ...

In Fillmore's theory, the hierarchy constrains the application of Subject Transformation. For example, if Patient is the subject, Instrument does not appear in the syntactic structure; hence, the sentence in (58) violates the hierarchy condition: 
(58) *The door opened with the key.

Our theory also predicts the ungrammaticality of (58). The unaccusative form of the verb corresponds to the conceptual structure representation [INCH (BE ([DOOR], [OPEN])])]. Neither Agent nor Instrument is present. (58) is ruled out because the instrumental phrase does not correspond to any semantic argument.

Along the same lines, the following sentence is ruled out:

(59) *The key opened the door by John.

The transitive form of the verb with the instrumental subject is associated with the conceptual structure in which the outermost CAUSE function and its first argument (i.e. Agent) is omitted; therefore, no Agent can be linked to the syntactic by phrase in (59). Note that Lasnik (1988) argues, primarily on the basis of the ungrammaticality of the sentence in question, that the theta-criterion should refer to the grammatical function, subject. However, this problem can be resolved on our approach without resorting to the grammatical role; thus, I see no reason for his suggesting an amendment to the theta-criterion.

Now consider the case where Instrument is linked to the by phrase as in the following:

(60) a. The door was opened by the skeleton key.

b. The horse was hit by the stick.

c. The wood was cut by the axe.

Suppose that the by phrase is linked to an external argument if it is demoted (see Grimshaw (1990)). It follows then that the Instruments realized as the by phrases in (60) are external. This means that the verbs correspond to the conceptual structure [CAUSE ([Z], [INCH ([BE ([Y], [ ])])])], where no Agent/Actor argument is involved. This account makes two predictions. First, if the Instrument is linked to the by phrase, the subject will not correspond to Agent because of the absence of the agent argument in the LCS:

(61) a. *John opened the door by the skeleton key.

b. *Elmer hit the horse by the stick.

Second, facilitating instruments are not realized as the by phrase because only demoted external arguments are linked to it. This prediction is born out by the following examples:

(62) a. *The inscription was examined by the magnifying glass.

b. *The thesis was written by a pen.

c. ${ }^{*}$ The horse was beaten by a stick. 
The claim that no Agent exists in conceptual structure if Instrument is linked to the by phrase raises a difficulty in explaining the presence of an "implicit Agent" in a sentence like (63):

(63) The ship was sunk by a torpedo [PRO to prove a point]. Apparently, the controller is not a torpedo. Under the standard view of implicit arguments, the sentence implies the presence of a syntactically unrealized argument at the level of argument structure, and it is this argument that controls the PRO. Since Agent is not present in the conceptual structure suggested on our account, the controller, whether implicit or not, does not exist. However, if we assume, along with Lasnik (1988), that the control involved in the above is actually "S-control", namely, the PRO is controlled by the matrix clause, the controller is not a particular argument of the verb but the ship was sunk by a torpedo. Adopting this idea, we may not posit an implicit Agent in argument structure.

\section{Concluding Remarks}

So far, we have discussed the problem of how the semantic dependency between action and instrumentality represented in verbs' LCS is manifested in syntactic structure. Our approach to this problem is essentially based on Jackendoff's semantic structure theory and Larson's theory of syntactic mapping of thematic roles.

It should be pointed out here that there is a substantial difference in their views as to the association of lexical semantic representations with syntactic representations. Larson's approach is based on the idea that a particular syntactic configuration is projected from the thematic structure specified by the meaning of a verb-the idea that appears in the recent theories of linking (See Rappaport and Levin (1988), Grimshaw (1990), Speas (1990)). By contrast, Jackendoff does not believe in the 'projection' hypothesis: his theory generates independent semantic representations and syntactic representations, which are linked through what he calls "correspondence rules". Although I have adopted Larson's approach in its essentials, I do not intend to reject Jackendoff's correspondence rule hypothesis. The methodological assumption underlying my account is that the mapping from conceptual relations to syntactic relations should be maximally simple and uniform.

Finally, I hope to have demonstrated in the present work that the distinction between argument and adjunct should be made at the level of 
LCS. A consequence of this claim is, I believe, that the linking theory needs no level of argument structure distinct from the LCS. In recent work (e.g. Rappaport and Levin (1988), Grimshaw (1990)), argument structure is actually devoid of thematic content. In Rappaport and Levin's version of argument structure, thematic roles are hierarchically represented, by means of variables to indicate the number of argument positions of a predicate. Similarly, in Grimshaw's theory, argument structure simply consists of a set of variables representing verb's arguments, which are ordered hierarchically as $(x(y(z)))$. However, given that thematic relations are defined in terms of argument positions in LCS and that there are some linking rules mapping semantic arguments onto syntactic relations, positing a separate level of argument structure would be more or less redundant since every theory has to have some means of accounting for linking regularities.

\section{REREFENCES}

Baker, Mark (1988a) Incorporation: A Theory of Grammatical Function Changing, University of Chicago Press, Chicago.

Baker, Mark (1988b) "Theta Theory and the Syntax of Applicatives in Chichewa," Natural Language and Linguistic Theory 6, 353-389.

Baker, Mark (1989) "Object Sharing and Projection in Serial Verb Constructions," Linguistic Inquiry 20, 513-553.

Bresnan, Joan and Jonni M. Kanerva (1989) "Locative Inversion in Chichewa: A

Case Study of Factorization in Grammar," Linguistic Inquiry 20, 1-50.

Bresnan, Joan and Lioba Moshi (1990) "Object Asymmetries in Comparative Bantu Syntax," Linguistic Inquiry 21, 147-185.

Chomsky, Noam (1986) Knowledge of Language: Its Nature, Origin, and Use, Praeger, New York.

Fillmore, Charles (1968) "The Case for Case," Universals in Linguistic Theory, ed. by Emmon Bach and Robert T. Harms, 1-90, Holt, Reinhart and Winston, New York. Foley, William A. and Robert D. Van Valin Jr. (1984) Functional Syntax and Universal Grammar, Cambridge University Press, Cambridge.

Grimshaw, Jane (1990) Argument Structure, MIT Press, Cambridge, MA.

Gropen, Jess, Steven Pinker, Michelle Hollander, and Richard Goldberg (1991)

"Affectedness and Direct Objects: The Role of Lexical Semantics in the

Acquisition of Verb Argument Structure," Cognition 41, 153-195.

Jackendoff, Ray (1983) Semantics and Cognition, MIT Press, Cambridge, MA. 
Jackendoff, Ray (1990) Semantic Structures, MIT Press, Cambridge, MA.

Larson, Richard K. (1988) "On the Double Object Construction," Linguistic Inquiry 19, 335-391.

Lasnik, Howard (1988) "Subjects and the $\theta$-Criterion," Natural Language and Linguistic Theory 6, 1-17.

Lebeaux, David (1988) "The Feature +Affected and the Formation of the Passive," Syntax and Semantics 21: Thematic Relations, ed. by Wendy Wilkins, 243-261, Academic Press, New York.

Levin, Beth and Malka Rappaport (1988) "Nonevent -er Nominals: A Probe into Argument Structure," Linguistics 26, 1067-1083.

Marantz, Alec (1984) On the Nature of Grammatical Relations, MIT Press, Cambridge, MA.

Pinker, Steven (1989) Learnability and Cognition: The Acquisition of Argument Structure, MIT Press, Cambridge, MA.

Rappaport, Malka and Beth Levin (1988) "What to Do with $\theta$-Roles," Syntax and

Semantics 21: Thematic Relations, ed. by Wendy Wilkins, 7-36, Academic Press, New York.

Ravin, Yael (1990) Lexical Semantics without Thematic Roles, Claredon Press, Oxford.

Schlesinger, Izchak M. (1989) "Instruments as Agents: On the Nature of Semantic Relations," Journal of Linguistics 25, 189-210.

Seiter, William J. (1979) "Instrumental Advancement in Niuean," Linguistic Inquiry 10, 595-621.

Speas, Margaret (1990) Phrase Structure in Natural Language, Kluwer, Dordrecht. Williams, Edwin (1981) “Argument Structure and Morphology," Linguistic Review $1,81-114$.

Department of English Naruto University of Education

Takashima, Naruto-shi

Tokushima, 772 